\title{
Cardioprotective effect of lipstatin derivative orlistat on normotensive rats submitted to cardiac ischemia and reperfusion ${ }^{1}$
}

Francisco Sandro Menezes-Rodrigues', Paolo Ruggero Errante", Regiane Miranda Ferreira", José Gustavo Padrão Tavares", Luciana de Paula"', Erisvaldo Amarante Araújo'v , Tânia Carmem Peñaranda

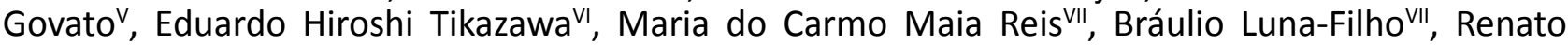
Ribeiro Nogueira Ferraz ${ }^{\mathrm{VIII}}$, Itamar de Souza Oliveira-Júnior ${ }^{\mathrm{IX}}$, Murched Omar TahalX, Afonso Caricati-Neto ${ }^{\mathrm{X}}$

'Fellow PhD degree, Postgraduate Program in Pharmacology, Universidade Federal de São Paulo (UNIFESP), Brazil. Conception and design of the study, analysis and interpretation of data, manuscript writing.

"Fellow PhD degree, Postgraduate Program in Pharmacology, UNIFESP, Sao Paulo-SP, Brazil. Pharmacological assays, analysis and interpretation of data.

"'Graduate student, Universidade Cidade de São Paulo (UNICID), Brazil. Biochemistry assays.

IVBSc, Faculades Metropolitanas Unidas (FMU), Sao Paulo-SP, Brazil. Statistical analysis.

${ }^{\vee}$ MSc, Assistant Professor, Department of Pharmacology, FMABC, Santo Andre-SP, Brazil. Pharmacological assays.

VIMD, Department of Public Health, Universidade de Campinas (UNICAMP), Brazil. Surgical procedures.

VIIPhD, Department of Medicine, UNIFESP, Sao Paulo-SP, Brazil. Interpretation of electrocardiogram.

VIIIFellow PhD degree, Postgraduate Program in Management of Health System (PMPA-GSS), Universidade Nove de Julho (UNINOVE), Sao Paulo-SP, Brazil. Critical revision.

${ }^{\mathrm{IX}}$ Associate Professor, Operative Technique and Experimental Surgery Division, Department of Surgery, UNIFESP, Sao Paulo-SP, Brazil. Critical revision.

${ }^{x}$ Associate Professor, Department of Pharmacology, UNIFESP, Sao Paulo-SP, Brazil. Conception and design of the study, critical revision.

\section{Abstract}

Purpose: To evaluate in vivo animal model of cardiac ischemia/reperfusion the cardioprotective activity of pancreatic lipase inhibitor of the orlistat.

Methods: Adult male Wistar rats were anesthetized, placed on mechanical ventilation and underwent surgery to induce cardiac I/R by obstructing left descending coronary artery followed by reperfusion to evaluation of ventricular arrhythmias (VA), atrioventricular block (AVB) and lethality (LET) with pancreatic lipase inhibitor orlistat (ORL). At the end of reperfusion, blood samples were collected for determination of triglycerides (TG), very lowdensity lipoprotein (VLDL), low-density lipoprotein (LDL), high-density lipoprotein (HDL), lactate dehydrogenase (LDH), creatine kinase (CK), and creatine kinase-MB (CK-MB).

Results: Treatment with ORL has been able to decrease the incidence of VA, AVB and LET. Besides that, treatment with ORL reduced serum concentrations of CK and LDL, but did not alter the levels of serum concentration of TG, VLDL and HDL.

Conclusion: The reduction of ventricular arrhythmias, atrioventricular block, and lethality and serum levels of creatine kinase produced by treatment with orlistat in animal model of cardiac isquemia/reperfusion injury suggest that ORL could be used as an efficient cardioprotective therapeutic strategy to attenuate myocardial damage related to acute myocardial infarction. Key words: Myocardial Infarction. Arrhythmias, Cardiac. Ischemia. Reperfusion. Biomarkers. Rats. 


\section{Introduction}

Acute myocardial infarction (AMI) is characterized by ischemic lesions that severely compromise the cardiac structure/ function, besides the survival of mammals. Although conventional therapy uses cardiac reperfusion, this procedure increases the cardiac damage caused by ischemia. The increasing prevalence of AMI, especially in the last decade, aroused the interest of pharmacologists in the search for compounds able to attenuate the cardiac damage by ischemia and reperfusion (I/R). These compounds have been classified as cardioprotective drugs. There are now numerous drug therapies available for use, such as lipid-lowering drugs (statins) that are being evaluated. The lipid-lowering effect of statins results from its inhibitory action on the enzyme regulating endogenous cholesterol synthesis. This action promotes the reduction of serum LDL concentration and the intensity of inflammatory processes mediated by $\mathrm{LDL}^{1-4}$.

The molecular mechanisms involved in cardioprotective activity of statins in patients with AMI remains unclear. The elevation of plasma triglycerides (TG) may indicate metabolic disorders, and its dosage is a parameter in the evaluation of arterial hypertension, diabetes, obesity and cardiaovascular risk. An elevation in plasma TG levels in patients with diabetes has been related with increased mobilization of lipid storages, increasing the risk of hepatic steatosis and atherosclerosis. However, the cardioprotective activity of lipid-lowering drugs that reduce pancreatic lipase (LP) activity, such as orlistat (ORL), in patients with $A M I$ is still poorly understood ${ }^{5,6}$.

Since hypertriglyceremia consists in an important risk factor for $\mathrm{AMI}$, in the present study, we decided to evaluate in vivo animal model of cardiac $\mathrm{I} / \mathrm{R}$ the cardioprotective activity of pancreatic lipase inhibitor of the ORL.

\section{Methods}

Animals were maintaned under standard conditions of nutrition, hydration, temperature, light and humidity, and in acordance to normatization approved by Ethical Committee of the EPM/UNIFESP (\#0065/12).

Adult male Wistar rats (14 - 16 weekold) weighting between 300 to $340 \mathrm{~g}$ were randomized into 4 groups: SHAM-operated $(n=10) ;$ cardiac $1 / R(n=40)$; treatment with vehicle $(I / R+V E, n=20)$ and treated with ORL for 10 days by gavage orally $(I / R+O R L$, $n=20$ ).

\section{Protocol of cardiac ischemia and reperfusion $(I / R)$}

It was used a method to induce cardiac I/R described by our lab ${ }^{7,8}$. Rats were anesthetized with urethane $(1.25 \mathrm{~g} / \mathrm{kg})$, and fixed in the supine position. After intubation (Jelco 14G, USA), rats were mechanically ventilated using a mechanic ventilator Insight model EFF 312 (Insight Equipamentos Científicos, Ribeirão Preto, Brazil). After stabilization for $15 \mathrm{~min}$, thoracotomy was performed to place the vascular tourniquet (4/0 braided silk suture attached to a $10-\mathrm{mm}$ micropoint reverse-cutting needle, Ethicon $\mathrm{K}-890 \mathrm{H}, \mathrm{USA})$ around the left anterior descending coronary artery to produce ischemia. After of $10 \mathrm{~min}$ of ischemia, the tourniquet was removed to allow coronary recirculation for $75 \mathrm{~min}$ (reperfusion).

The cardiac electrical activity in SHAM-operated and cardiac I/R groups was 
monitorated by electrocardiogram (ECG) system using a method to described by our $\mathrm{lab}^{7,8}$. ECG evaluation was performed during all experiment (100 min duration). The ECG was recorded using a biopotential amplifier by means of needle electrodes placed subcutaneously on the limbs ${ }^{7,8}$. Successful surgical obstruction of the coronary artery was valided by ECG alterations (increase in $R$ wave and ST segment) caused by ischemia ${ }^{7,8}$. The body temperature was mantained at 37.5 으 with a heated operating platform and appropriate heating lamps, and was evaluated routinely via a rectal thermometer.

\section{ECG analysis}

The ECG data were recorded using an acquisition system AqDados 7.02 (Lynx Tecnologia Ltda., Brazil), and analysed using the software AqDAnalysis 7 (Lynx Tecnologia Ltda., Brazil). Using this software, were evaluated the heart rates, as well as incidence of ventricular arrhythmias (VA), atrioventricular block (AVB) and lethality (LET), in response to cardiac I/R. The ventricular fibrillation, torsades de pointes, and ventricular tachycardia parameters were considered only as VA. After ECG recording, blood sample were collected from the abdominal aorta artery, and centrifuged $(2,500$ rpm, for $40 \mathrm{~min}$, at $5^{\circ} \mathrm{C}$ ) to isolation of serum. The serum was stored at $-20^{\circ} \mathrm{C}$ for biochemical analysis.

\section{Biochemical parameters}

For determination of triglycerides by lipoprotein lipase (LPL) enzyme activity in serum, it was performed by measuring enzymatic colorimetric test, measured at $505 \mathrm{~nm}$ (Kit VIDA Biotecnologia, Belo Horizonte, Brazil). For determination of serum cholesterol, it was performed by colorimetric test, measured at $505 \mathrm{~nm}$ (Kit VIDA Biotecnologia, Belo Horizonte, Brazil). For the quantitative determination of the HDL fraction of Cholesterol in serum, it was performed through Enzyme/Colorimetric test, measured at 570 to $610 \mathrm{~nm}$ (Kit VIDA Biotecnologia, Belo Horizonte, Brazil).

Quantitative determination of lactic dehydrogenase $(\mathrm{LDH})$, creatine kinase $(\mathrm{CK})$, and creatine kinase $\mathrm{MB}$ fraction (CK-MB) was performed using a kinetic-UV method, measured at $340 \mathrm{~nm}$ (Kit Katal Biotecnológica Ind. Com. Ltda., Belo Horizonte, Brazil).

\section{Data analysis}

Statistical analysis of incidence of VA, AVB, and LET were statistically evaluated using the Fisher's exact test $(p<0.05)$. Statistical analysis of biomarkers of cardiac injury were expressed as mean \pm standard error (SE) of the mean, and analysis of variance (ANOVA) was applied, followed by the Tukey posttest ( $p<0.05)$. These statistical analysis were performed using Prism 5.0 software (GraphPad, USA).

\section{Results}

Our results showed that treatment with ORL decreased the percentual incidence of VA $(85 \%$ to $57 \%$, Figure $1 \mathrm{~A})$, AVB $(79 \%$ to $43 \%$, Figure $1 B$ ), and LET ( $70 \%$ to $38 \%$, Figure $1 \mathrm{C})$ in animals. 

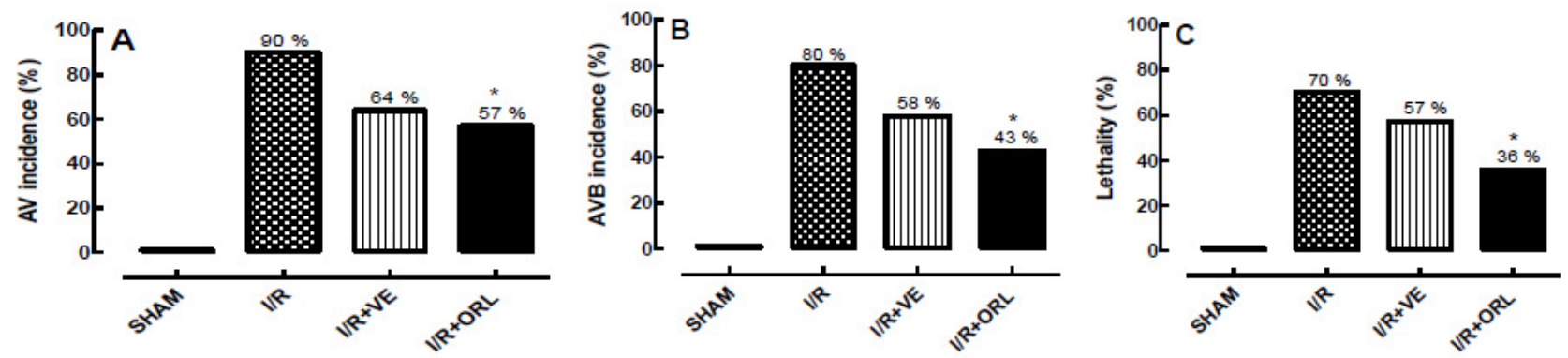

Figure 1 - (A) Incidence of the ventricular arrhythmias (AV). (B) Atrioventricular block (AVB). (C) Lethality (LET) in the SHAM, I/R, I/R+VE and I/R+ORL groups. The results were expressed as mean, and Fisher's exact test $\left({ }^{*} p<0.05\right)$. A reduced of incidence of the AV, AVB and LET was observed the group of $\mathrm{I} / \mathrm{R}+\mathrm{ORL}$ groups when compared with $\mathrm{I} / \mathrm{R}$ group. SHAM= simulated group; $\mathrm{I} / \mathrm{R}=$ cardiac ischemia and reperfusion group; $\mathrm{I} / \mathrm{R}+\mathrm{VE}=$ cardiac ischemia and reperfusion group treated with vehicle; $I / R+O R L=$ cardiac ischemia and reperfusion group treated with orlistat. *Statistically different from the cardiac I/R and SHAM groups.

The treatment with $\mathrm{ORL}$ reduced the serum concentrations of the LDL $(40.9 \pm 4.8$ to $10.2 \pm 2.7 \mathrm{mg} / \mathrm{dL}$, Figure $2 \mathrm{C})$ and CK (4564.8 \pm 470.4 to $2387.2 \pm 306.7 \mathrm{U} / \mathrm{L}$, Figure $3 \mathrm{~B}$ ) in animals. In contrast, the ORL effect in I/R group did not alter the levels of serum concentration of the of TG $(101.7 \pm 7.12 \mathrm{mg} / \mathrm{dL}$, Figure $2 \mathrm{~A})$, $\operatorname{VLDL}(21.38 \pm 1.60 \mathrm{mg} / \mathrm{dL}$, Figure $2 \mathrm{~B})$, and HDL $(35.00 \pm 5.01 \mathrm{mg} / \mathrm{dL}$, Figure $2 \mathrm{D})$, such as of the LDH $(1653.0 \pm 91.9$, Figure $3 \mathrm{~A})$ and CK-MB (1849.8 U/L \pm 221.9 , Figure $3 C$ ) in animals.
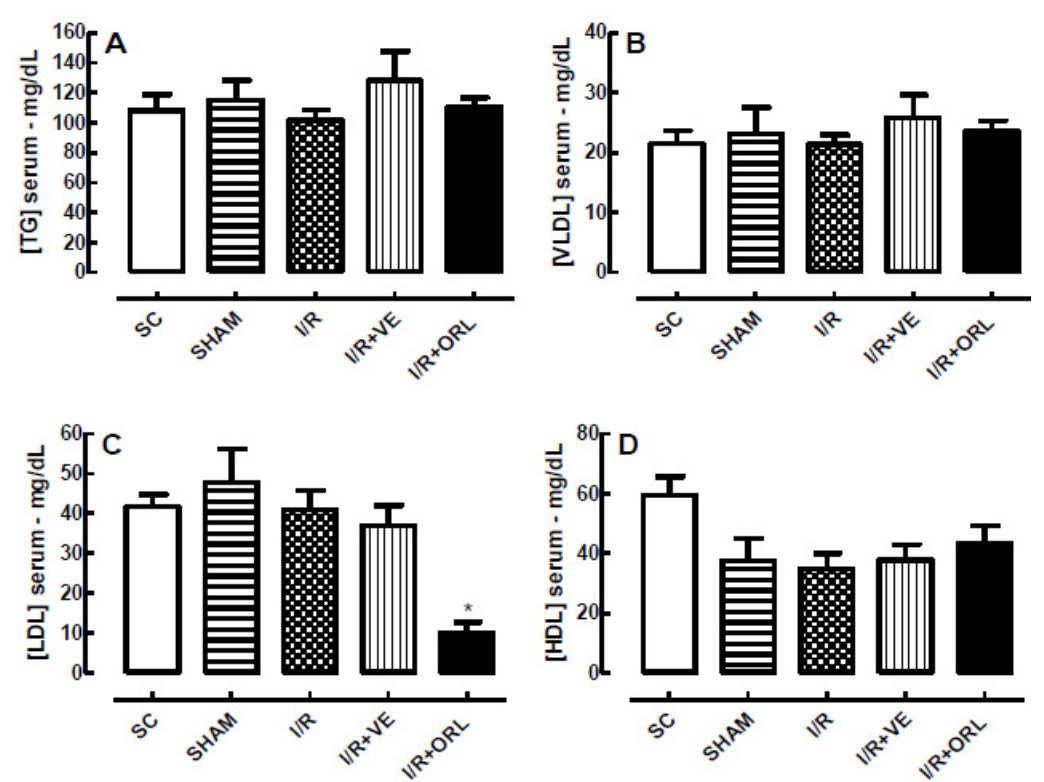

Figure 2 - Serum concentration of TG (A), VLDL (B), LDL (C) and HDL (D) in the SC, SHAM, I/R, I/R+VE and I/ $\mathrm{R}+\mathrm{ORL}$ groups. The results were expressed as mean \pm standard error of the mean, and analysis of variance (ANOVA) was applied, followed by the Tukey post-test $\left({ }^{*} p<0.05\right) . S C=$ group without surgery; SHAM= simulated group; $\mathrm{I} / \mathrm{R}=$ cardiac ischemia and reperfusion group; $\mathrm{I} / \mathrm{R}+\mathrm{VE}=$ cardiac ischemia and reperfusion group treated with vehicle; $\mathrm{I} / \mathrm{R}+\mathrm{ORL}=$ cardiac ischemia and reperfusion group treated with orlistat. *Statistically different from all other groups. 

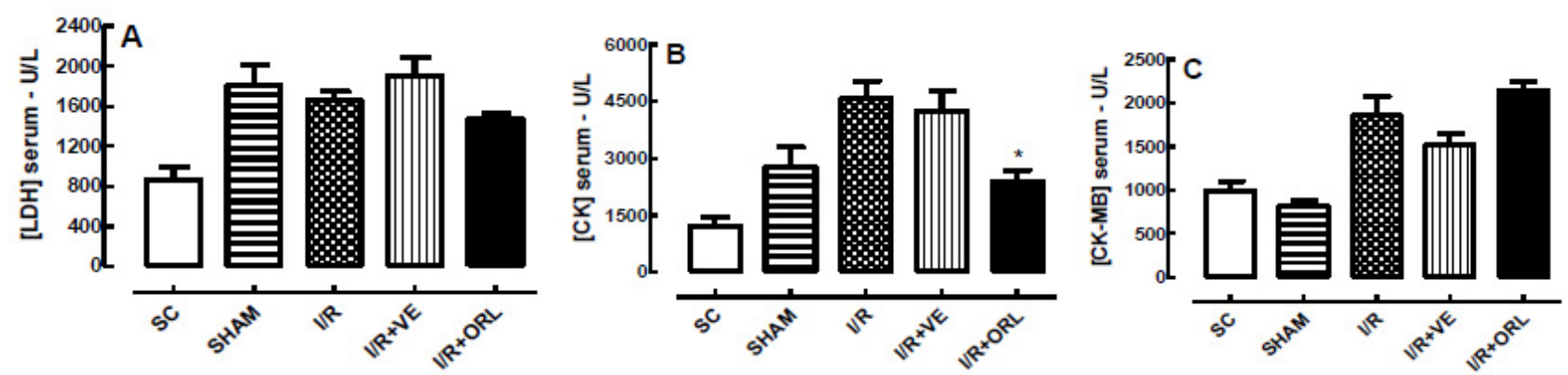

Figure 3 - Serum concentration of LDH (A), CK (B) and CK-MB (C) in the SC, SHAM, $1 / R, I / R+V E$ and I/R+ORL groups. The results were expressed as mean \pm standard error of the mean, and analysis of variance (ANOVA) was applied, followed by the Tukey post-test $\left({ }^{*} p<0.05\right)$. SC= group without surgery; SHAM= simulated group; $\mathrm{I} / \mathrm{R}=$ cardiac ischemia and reperfusion group; $\mathrm{I} / \mathrm{R}+\mathrm{VE}=$ cardiac ischemia and reperfusion group treated with vehicle; $\mathrm{I} / \mathrm{R}+\mathrm{ORL}=$ cardiac ischemia and reperfusion group treated with orlistat. *Statistically different from the cardiac I/R group.

\section{- Discussion}

Ours results showed that treatment of animals submitted to cardiac I/R for 10 days with the pancreatic lipase inhibitor ORL reduced the incidence of $V A, A V B$ and LET, as well as decreased serum levels of $C K$ and LDL-cholesterol.

The metabolic syndrome is a complex disorder represented by a set of cardiovascular risk factors usually related to central fat deposition and insulin resistance. It is important to highlight the association of metabolic syndrome with cardiovascular disease, increasing overall mortality by 1.5 times and cardiovascular mortality by 2.5 times ${ }^{9-13}$. In this situation, dyslipidemia is characterized by the presence of high levels of LDL-cholesterol and low levels of HDL-cholesterol, and elevated levels of triglycerides ${ }^{2}$. The drugs capable of reducing the absorption of lipids such as cholesterol may interfere with vascular inflammatory processes, like ezetimibe, a selective inhibitor of intestinal cholesterol absorption by the cholesterol carrier Niemann-Pick C1 Like 1 protein. The ezetimibe is able to block vascular inflammation triggered by oxidative stress, by decreasing serum LDL concentrations, synthesis of monocyte attraction protein-1 chemokine and the expression of the leukocyte-to-endothelial cell adhesion, such as VCAM-1 and transcriptional synthesis of messenger RNA for CD14 ${ }^{14}$.

Elevation of plasma TG may indicate metabolic disorders, and its dosage is a parameter in the evaluation of uremia, diabetes, obesity and cardiac risk. An elevation in plasma TG levels in patients with diabetes is related to increased mobilization of lipid storage areas, increasing the risk of hepatic steatosis and atherosclerosis ${ }^{15}$.

The ORL binds covalently to the serine portion of the site of action of lipases causing irreversible inhibition of gastrointestinal lipases (gastric and pancreatic lipase) ${ }^{5,6}$. ORL is a drug partially derived from an endogenous lipstatin produced by Streptomyces toxytricini, and inhibits the pancreatic lipase enzyme, responsible for the hydrolysis of TG ingested from the diet ${ }^{5,6}$.

When administrated orally, approximately 1 hour before the three main meals, the ORL reduces dietary fat absorption by $30 \%{ }^{16}$ and is used in association with a diet for the treatment of obesity in patients with 
body mass index (BMI) greater than or equal to $30 \mathrm{~kg} / \mathrm{m}^{2}$, or in patients with excess body mass (BMI greater than or equal to $28 \mathrm{~kg} / \mathrm{m}^{2}$ ) who are at risk of disease.

Since hypertriglycerectomy is a risk factor for AMI, we decided to quantify TG plasma concentration in animals and to correlate it with the electrophysiological and cardiac changes in our $I / R$ model. The $I / R$ protocol did not cause a statistically significant change in the TG concentration. Similarly, treatment of rats with ORL was also unable to modify the serum TG value observed in the $I / R$ group. Primary dyslipidemias, such as common hypertriglyceridemia, familial hypertriglyceridemia, combined familial hyperlipidemia, are less important epidemiologically when compared to secondary dyslipidemias observed in diabetes mellitus, obesity and alcoholism, or related to the use of medications such as diuretics, betablockers deprived of ASI, contraceptives and corticosteroids ${ }^{17}$.

The TG is considered important biological markers for other lipid alterations with atherogenic potential, and may indicate alterations such as combined familial hyperlipidemia, decrease in HDL levels and diabetes mellitus. Also, increased serum TG levels increase the risk of thrombogenesis when interacting with coagulation factors and platelets ${ }^{13}$, and its association with other risk factors such as hypertension, smoking, diabetes mellitus, morbid obesity and postmenopausal, increase morbidity and mortality, especially in the case of ischemic heart diseases ${ }^{17}$.

The use of $120 \mathrm{mg}$ of ORL (after 2 hours of ingestion) caused decrease in serum TG levels in overweight and type 2 diabetic patients ${ }^{15}$ or in serum chylomicrons 8 hours after use of ORL ${ }^{18}$, as a function of decreased intestinal absorption. The use of short-term
ORL caused decrease in serum TG levels in patients with familial hypercholesterolemia ${ }^{19}$, as well as the use during 6 months in obese patients ${ }^{1,20}$, or for 1 year in obese patients, obese with hypercholesterolemia ${ }^{7}$ or obese with type 2 diabetes ${ }^{21}$, which favors the reduction the use of oral hypoglycemic agents and insulin. Also, the combination of ORL with Genfibrozil ${ }^{22}$ or Statin ${ }^{23}$ reduced triglyceride levels by $35 \%$ in patients with hypertriglyceridemia.

The ORL in combination with a hypocaloric diet, reduces serum TG levels, an effect associated with a decrease in the intestinal absorption of triglycerides by ORL and widely described in the literature ${ }^{4,24,25}$, but this effect was not observed in our study.

Although the elevation of LDL cholesterol is not considered one of the diagnostic criteria for the metabolic syndrome, patients with this syndrome present alterations in the density and particle size of the lipoprotein, predominantly the type $B$ pattern (small and dense LDL). This association is termed atherogenic dyslipidemia. Patients with metabolic syndrome have a high risk of cardiovascular disease when compared to those without metabolic syndrome, and the two first-line medications in the treatment of obesity associated with the metabolic syndrome are sibutramine and ORL ${ }^{16}$.

Biomarkers of myocardial injury have been studied since the 1970s, among them the LDH enzyme ${ }^{26}$. In our study, no change in LDH levels was observed, and others studies using orlistat not have found beneficial effect on the decrease of $\mathrm{LDH}^{14,20}$. The CK constitutes another important biomarker for the diagnosis of AMI, which is an enzyme that regulates the production and use of high energy phosphate in the contractile tissues, like myocardium. The determination of serum levels of total CK and myocardial CK (CK-MB) is important to 
diagnosis of AMI.

In our study, we observed a statistical difference in the reduction levels of $\mathrm{CK}$; this may due to the action of ORL on endothelial function and LDL reduction, promoting cardiovascular benefits, and cardioprotection against the $\mathrm{I} / \mathrm{R}^{25,27}$. Zhi et al. ${ }^{28}$ demonstrated by screening that there is an extremely low degree of systemic absorption for orlistat when administered with a hypocaloric, with small levels in plasma and urine. Therefore, we believe that in spite of presenting a low systemic bioavailability, we propose that ORL after reaching plasma promotes inhibition of the lipoprotein lipase enzyme produced by vasculature from skeletal muscles and adipose tissue, thus exerting in addition to local effects on the gastrointestinal tract and systemic effects.

The treatment with ORL was not reversed the increase in serum CK-MB when compared with I/R group. In the literature was not reported association between the use of the orlist and dosage of CK or CK-MB.

The reduction of $V A, A V B$ and $L E T$, and serum levels of CK and LDL produced by treatment during 10 days with pancreatic lipase inhibitor ORL in animals submitted to cardiac I/R injury clearly indicate the cardioprotective activity of ORL.

\section{- Conclusions}

The reduction of ventricular arrhythmias, atrioventricular block, and lethality and serum levels of creatine kinase produced by treatment with orlistat in animal model of cardiac isquemia/reperfusion injury suggest that ORL could be used as an efficient cardioprotective therapeutic strategy to attenuate myocardial damage related to acute myocardial infarction.

\section{References}

1. Audikovszky M, Pados G, Seres I, Harangi M, Fülöp $P$, Katona E, Illyés L, Winkler G, Katona EM, Paragh G. Orlistat increases serum paraoxonase activity in obese patients. Nutr Metab Cardiovasc Dis. 2007;17(4):268-73. doi: 10.1016/j.numecd.2006.03.004.

2. Austin MA, Breslow JL, Hennekens $\mathrm{CH}$, Buring JE, Willett WC, Krauss RM. Lowdensity lipoprotein subclass patterns and risk of myocardial infarction. JAMA. 1988;260(13):1917-21. doi: 10.1001/ jama.1988.03410130125037.

3. Bers DM. Calcium cycling and signaling in cardiac myocytes. Annu Rev Physiol. 2008;70:23-49. doi: 10.1146/annurev. physiol.70.113006.100455.

4. Bloch KV, Salles GF, Muxfeldt ES, da Rocha Nogueira A. Orlistat in hypertensive overweight/obese patients: results of a randomized clinical trial. J Hypertens. 2003;21(11):2159-65. doi: 10.1097/01. hjh.0000098137.70956.8c.

5. Cheah JS. Orlistat (Xenical) in the management of obesity. Ann Acad Med Singapore. 2000;29(4):419-20. PMID: 11056767.

6. Crenier L, Sternon J. Orlistat (Xenical). Rev Med Brux. 1999;20(3):159-63. PMID: 10429540.

7. TavaresJGP, Vasques ER, Arida RM, Cavalheiro EA, Cabral FR, Torres LB, Menezes-Rodrigues FS, Jurkiewicz A, Caricati-Neto A, Godoy CM, Gomes da Silva S. Epilepsy-induced electrocardiographic alterations following cardiac ischemia and reperfusion in rats. Braz J Med Biol Res. 2015;48(2):140-5. doi: 10.1590/1414-431X20144311.

8. Tavares JGP, Menezes-Rodrigues FS, Vasques $E R$, Reis MCM, Paula Luna-Filho B, Errante PR, Caricati-Neto A, Bergantin LB. A simple and efficient methodology for the study of cardioprotective drugs in animal model of cardiac ischemia-reperfusion. J Mol Imag Dynamic. 2017;7(133):2. doi: 10.4172/21559937.10001.

9. Ford ES, Giles WH. A comparison of the prevalence of the metabolic syndrome using two proposed definitions. Diabetes Care. 2003;26(3):575-81. doi: 10.2337/ diacare.26.3.575. 
10.Hu G, Qiao Q, Tuomilehto J, Balkau B, Borch-Johnsen K, Pyorala K. Prevalence of the metabolic syndrome and its relation to all-cause and cardiovascular mortality in nondiabetic European men and women. Arch Int Med. 2004;164(10):1066-76. doi: 10.1001/archinte.164.10.1066.

11. Girman CJ, Rhodes T, Mercuri M, Pyörälä K, Kjekshus J, Pedersen TR, Beere PA, Gotto AM, Clearfield M; 4S Group and the AFCAPS/ TexCAPS Research Group. The metabolic syndrome and risk of major coronary events in the Scandinavian Simvastatin Survival Study (4S) and the Air Force/Texas coronary atherosclerosis prevention study (AFCAPS/ TexCAPS). Am J Cardiol. 2004;93(2):136-41. doi: 10.1016/j.amjcard.2003.09.028.

12. Haffner S, Taegtmeyer H. Epidemic obesity and the metabolic syndrome. Circulation. 2003;108(13):1541-5. doi: 0.1161/01. CIR.0000088845.17586.EC.

13.Lakka H-M, Laaksonen DE, LakkaTA, Niskanen LK, Kumpusalo E, Tuomilehto J, Salonen JT. The metabolic syndrome and total and cardiovascular disease mortality in middleaged men. JAMA. 2002;288(21):2709-16. doi: 10.1001/jama.288.21.2709.

14.Gómez-Garre D, Muñoz-Pacheco $P$, González-Rubio ML, Aragoncillo $P$, Granados R, Fernández-Cruz A. Ezetimibe reduces plaque inflammation in a rabbit model of atherosclerosis and inhibits monocyte migration in addition to its lipid-lowering effect. $\mathrm{Br} J$ Pharmacol. 2009;156(8):1218-27. doi: 10.1111/j.14765381.2008.00091.x.

15.Tan KCB, Tso AWK, Tam SCF, Pang RWC, Lam KSL. Acute effect of orlistat on postprandial lipaemia and free fatty acids in overweight patients with Type 2 diabetes mellitus. Diabet Med. 2002;19(11):944-8. doi: 10.1046/j.1464-5491.2002.00823.x.

16. Halpern A, Mancini MC. Treatment of obesity: an update on anti-obesity medications. Obes Rev. 2003;4(1):25-42 doi: 10.1046/j.1467-789X.2003.00083.x

17.Sociedade Brasileira de Cardiologia. Consensos/Diretrizes. Disponível em: http:// publicacoes.cardiol.br/2014/diretrizes.asp.

18. Reitsma JB, Cabezas MC, de Bruin TW, Erkelens DW. Relationship between improved postprandial lipemia and lowdensity lipoprotein metabolism during treatment with tetrahydrolipstatin, a pancreatic lipase inhibitor. Metabolism. 1994;43(3):293-8. doi: 10.1016/00260495(94)90095-7.

19.Tzotzas T, Krassas GE, Bruckert E. Administration of orlistat in a patient with familial hyperchylomicronemia. Atherosclerosis. 2002;165(1):185-6. PMID: 12208486.

20.Gokcell A, Gumurdulu Y, Karakosel H. Evaluation of safety and efficacy of sibutramine, orlistat and metformin in the treatment of obesity with diabetes. Diabetes Obes Metab. 2022;4(1):49-55. PMID: 11874442.

21. Hollander PA, Elbein SC, Hirsch IB, Kelley D, McGill J, Taylor T, Weiss SR, Crockett SE, Kaplan RA, Comstock J, Lucas CP, Lodewick PA, Canovatchel W, Chung J, Hauptman J. Role of orlistat in the treatment of obese patients with type 2 diabetes: a 1-year randomized double-blind study. Diabetes Care. 1998;21(8):1288-94. doi: 10.2337/ diacare.21.8.1288.

22. Tolentino MC, Ferenczi A, Ronen L, Poretsky L. Combination of gemfibrozil and orlistat for treatment of combined hyperlipidemia with predominant hypertriglyceridemia. Endocr Pract. 2002;8(3):208-12. doi: 10.4158/ EP.8.3.208.

23.Wierzbicki AS, Reynolds TM, Crook MA. Usefulness of Orlistat in the treatment of severe hypertriglyceridemia. Am J Cardiol. 2002;89(2):229-31. PMID: 11792350.

24.Kiortsis DN, Filippatos TD, Elisaf MS. The effects of orlistat on metabolic parameters and other cardiovascular risk factors. Diabetes Metab. 2005;31(1):15-22. doi: 10.1016/S1262-3636(07)70161-1.

25.Turker I, Demirag NG, Tanaci N, Tutar $\mathrm{NU}$, Kirbas I. Effects of orlistat plus diet on postprandial lipemia and brachial artery reactivity in normolipidemic, obese women with normal glucose tolerance: a prospective, randomized, controlled study. Curr Ther Res Clin Exp. 2006;67(3):159-73. doi: 10.1016/j.curtheres.2006.06.001.

26.Norris JW, Hachinski VC, Myers MG, Callow J, Wong T, Moore RW. Serum cardiac enzymes in stroke. Stroke. 1979;10(5):548-53. doi: 10.1161/01.STR.10.5.548.

27.Yu CC, Li AM, Chan KO, Chook P, Kam JT, Au $C T$, So RC, Sung RY, McManus AM. Orlistat 
improves endothelial function in obese adolescents: a randomised trial. J Paediatr Child Health. 2013;49(11):969-75. doi: 10.1111/jpc.12252.

28.Zhi J, Melia AT, Eggers H, Joly R, Patel IH.
Review of limited systemic absorption of orlistat, a lipase inhibitor, in healthy human volunteers. J Clin Pharmacol. 1995;35(11):1103-8. doi: 10.1002/j.15524604.1995.tb04034.x.

\section{Correspondence:}

Afonso Caricati Neto

Laboratório de Farmacologia Autonômica e Cardiovascular, UNIFESP

Rua Pedro de Toledo, 699/7ㅇ andar

04039-032 São Paulo - SP Brasil

caricatineto@gmail.com

Received: Feb 09, 2018

Review: Apr 08, 2018

Accepted: May 12, 2018
Conflict of interest: none

Financial sources: CNPq, FAPESP and CAPES

${ }^{1}$ Research performed at Laboratório de Farmacologia Autonômica e Cardiovascular (LabFAC), Department of Pharmacology, Universidade Federal de São Paulo (UNIFESP), Brazil. Part of PhD thesis, Postgraduate Program in Pharmacology. Tutor: Prof. Dr. Afonso Caricati-Neto. 


\title{
Erratum
}

\author{
Publication: Acta Cir Bras. vol.33 no.6 Sao Paulo June 2018
}

http://dx.doi.org/10.1590/s0102-865020180060000007

\section{ORIGINAL ARTICLES}

\section{7 - Experimental Surgery}

MANUSCRIPT: Cardioprotective effect of lipstatin derivative orlistat on normotensive rats submitted to cardiac ischemia and reperfusion ${ }^{1}$

Francisco Sandro Menezes-Rodrigues', Paolo Ruggero Errante", Regiane Miranda Ferreira", José Gustavo Padrão Tavares", Luciana de Paula"I", Erisvaldo Amarante Araújo'v, Tânia Carmem Peñaranda

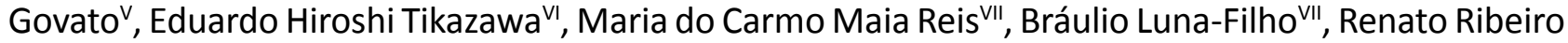
Nogueira FerrazIIIII, Itamar de Souza Oliveira-Júnior'x, Murched Omar Taha' ${ }^{1 \mathrm{X}}$, Afonso Caricati-Neto ${ }^{\mathrm{X}}$

'Fellow PhD degree, Postgraduate Program in Pharmacology, Universidade Federal de São Paulo (UNIFESP), Brazil. Conception and design of the study, analysis and interpretation of data, manuscript writing.

"Fellow PhD degree, Postgraduate Program in Pharmacology, UNIFESP, Sao Paulo-SP, Brazil. Pharmacological assays, analysis and interpretation of data.

"'Graduate student, Universidade Cidade de São Paulo (UNICID), Brazil. Biochemistry assays.

IVBSc, Faculades Metropolitanas Unidas (FMU), Sao Paulo-SP, Brazil. Statistical analysis.

${ }^{\vee} \mathrm{MSc}$, Assistant Professor, Department of Pharmacology, FMABC, Santo Andre-SP, Brazil. Pharmacological assays.

${ }^{V} M D$, Department of Public Health, Universidade de Campinas (UNICAMP), Brazil. Surgical procedures.

VIIPhD, Department of Medicine, UNIFESP, Sao Paulo-SP, Brazil. Interpretation of electrocardiogram.

VIIIFellow PhD degree, Postgraduate Program in Management of Health System (PMPA-GSS), Universidade Nove de Julho (UNINOVE), Sao Paulo-SP, Brazil. Critical revision.

${ }^{\mathrm{IX}}$ Associate Professor, Operative Technique and Experimental Surgery Division, Department of Surgery, UNIFESP, Sao Paulo-SP, Brazil. Critical revision.

${ }^{x}$ Associate Professor, Department of Pharmacology, UNIFESP, Sao Paulo-SP, Brazil. Conception and design of the study, critical revision.

Where was it read:

Itamar de Souza Oliveira-Júnior ${ }^{1 \mathrm{x}}$

Read:

Itamar Souza de Oliveira-Júnior

Where was it read:

${ }^{1 x}$ Associate Professor, Operative Technique and Experimental Surgery Division, Department of Surgery, UNIFESP, Sao Paulo-SP, Brazil. Critical revision.

Read:

${ }^{1 X}$ Associate Professor, Anesthesia, Pain and Intensive Medicine Division, Department of Surgery, UNIFESP, Sao Paulo-SP, Brazil. Critical revision. 